\title{
UNIVALENCY OF CONVOLUTIONS OF UNIVALENT HARMONIC RIGHT HALF-PLANE MAPPINGS
}

\author{
ZHIHONG LIU AND SAMINATHAN PONNUSAMY
}

\begin{abstract}
We consider the convolution of half-plane harmonic mappings with respective dilatations $(z+a) /(1+a z)$ and $e^{i \theta} z^{n}$, where $-1<a<1$ and $\theta \in \mathbb{R}, n \in \mathbb{N}$. We prove that such convolutions are locally univalent for $n=1$, which solves an open problem of Dorff et. al (see [3, Problem 3.26]). Moreover, we provide some numerical computations to illustrate that such convolutions are not univalent for $n \geq 2$.
\end{abstract}

\section{Introduction AND PRELIMINARIES}

The subject of this study is the convolution of functions in the class $\mathcal{H}$ of all complex-valued harmonic mappings $f=h+\bar{g}$ defined on the open unit disk $\mathbb{D}=\{z \in \mathbb{C}:|z|<1\}$ normalized by the condition $h(0)=g(0)=h^{\prime}(0)-1=0$, where $h$ and $g$ are analytic in $\mathbb{D}$. For two such harmonic mappings $f$ and $F=H+\bar{G}$ in $\mathcal{H}$ with power series of the form

$$
f(z)=z+\sum_{n=2}^{\infty} a_{n} z^{n}+\sum_{n=1}^{\infty} \overline{b_{n}} \bar{z}^{n} \text { and } F(z)=z+\sum_{n=2}^{\infty} A_{n} z^{n}+\sum_{n=1}^{\infty} \overline{B_{n}} \bar{z}^{n},
$$

we define the harmonic convolution (or Hadamard product) as follows:

$$
f * F=h * H+\overline{g * G}=z+\sum_{n=2}^{\infty} a_{n} A_{n} z^{n}+\sum_{n=1}^{\infty} \overline{b_{n} B_{n}} \bar{z}^{n} .
$$

By Lewy's theorem [12], $f \in \mathcal{H}$ is locally univalent and sense-preserving if and only if $J_{f}(z)>0$ in $\mathbb{D}$, where $J_{f}(z)=\left|h^{\prime}(z)\right|^{2}-\left|g^{\prime}(z)\right|^{2}$ denotes the Jacobian of $f$. The condition $J_{f}(z)>0$ is equivalent to the existence of an analytic function $\omega$, called the dilatation of $f$, given by $\omega(z)=g^{\prime}(z) / h^{\prime}(z)$ with $|\omega(z)|<1$ for all $z \in \mathbb{D}$, where $h^{\prime}(z) \neq 0$ in $\mathbb{D}$. Denote by $\mathcal{S}_{H}$ the class of all sense-preserving harmonic univalent mappings $f=h+\bar{g} \in \mathcal{H}$ and by $\mathcal{S}_{H}^{0}$ the subclass of mappings $f \in \mathcal{S}_{H}$ such that $f_{\bar{z}}(0)=0$. For many basic results about several geometric subclasses of $\mathcal{S}_{H}$ and $\mathcal{S}_{H}^{0}$, we refer to the article of Clunie and Sheil-Small [4] (or see [7, 9, 16]). Denote by $\mathcal{K}_{H}^{0}$, the class of functions in $\mathcal{S}_{H}^{0}$ which have convex images, and functions in $\mathcal{K}_{H}^{0}$ are called convex.

Unlike the case of analytic mappings, the properties of harmonic convolutions are not so regular in the sense that the convolution of two convex harmonic mappings is not necessarily even locally univalent in $\mathbb{D}$. However, these convolutions do exhibit some fascinating properties. In recent years, properties of convolutions of harmonic mappings were investigated by a number of authors, see for example [6, 8, 13, 15, 18] and the references therein. In [1, 5] and [10], explicit descriptions of half-plane mappings and strip mappings are given.

Recall that a domain $\Omega \subset \mathbb{C}$ is said to be convex in the horizontal direction (CHD) if the intersection of $\Omega$ with each horizontal line is connected (or empty). We now recall one of the fundamental results, called shearing theorem, due to Clunie and Sheil-Small [4].

Theorem A. Let $f=h+\bar{g}$ be harmonic and locally univalent in $\mathbb{D}$. Then $f$ is univalent and its range is $\mathrm{CHD}$ if and only if $h-g$ is univalent and its range is $\mathrm{CHD}$.

2010 Mathematics Subject Classification. 31A05, 30C45.

Key words and phrases. Convolution, univalent harmonic mappings, right half-plane mappings, convex in the horizontal direction. 
A function $f=h+\bar{g} \in \mathcal{S}_{H}$ is said to be a slanted half-plane mapping with $\gamma(0 \leq \gamma<2 \pi)$ if $f$ maps $\mathbb{D}$ onto $H_{\gamma}:=\left\{w: \operatorname{Re}\left(e^{i \gamma} w\right)>-(1+a) / 2\right\}$, where $-1<a<1$. Using the shearing method due to Clunie and Sheil-Small [4] and the Riemann mapping theorem, it is easy to see that such a mapping has the form

$$
h(z)+e^{-2 i \gamma} g(z)=\frac{(1+a) z}{1-e^{i \gamma} z} .
$$

Note that $h(0)=g(0)=h^{\prime}(0)-1=0$ and $g^{\prime}(0)=a$. The class of all slanted half-plane mappings with $\gamma$ is denoted by $\mathcal{S}\left(H_{\gamma}\right)$. Clearly, each $f \in \mathcal{S}\left(H_{\gamma}\right)$ obviously belongs to the convex family $\mathcal{K}_{H}$ but not necessarily in $\mathcal{K}_{H}^{0}$ unless $a=0$. It is evident that there are infinitely many slanted half-plane mappings with a fixed $\gamma$. We denote by $\mathcal{S}^{0}\left(H_{\gamma}\right)$ if $a \in(-1,1)$ in $\mathcal{S}\left(H_{\gamma}\right)$ is taken to be zero. At this point, it is worth recalling that functions $f=h+\bar{g} \in \mathcal{S}^{0}\left(H_{0}\right)$ (i.e. $f \in \mathcal{S}\left(H_{\gamma}\right)$ with $a=0$ ) are usually referred to as the slanted half-plane mappings with $\gamma$ and such mappings by (1.1) obviously assume the form

$$
h(z)+e^{-2 i \gamma} g(z)=\frac{z}{1-e^{i \gamma} z}
$$

so that $\gamma=0$ reduces to the corresponding right half-plane mappings. For example, if $f_{0}=h_{0}+\overline{g_{0}} \in$ $\mathcal{S}^{0}\left(H_{0}\right)$ with the dilatation $\omega_{0}=g_{0}^{\prime} / h_{0}^{\prime}=-z$ then the shearing theorem of Clunie and Sheil-Small quickly yields (see [4])

$$
h_{0}=\frac{z-\frac{1}{2} z^{2}}{(1-z)^{2}} \quad \text { and } \quad g_{0}=\frac{-\frac{1}{2} z^{2}}{(1-z)^{2}} .
$$

The function $f_{0}$ is extremal for the coefficient inequality for functions in $\mathcal{K}_{H}^{0}$.

Theorem B. ([8, Theorem 2]) If $f_{k} \in \mathcal{S}^{0}\left(H_{\gamma_{k}}\right), k=1,2$, and $f_{1} * f_{2}$ is locally univalent in $\mathbb{D}$, then $f_{1} * f_{2}$ is convex in the direction $-\left(\gamma_{1}+\gamma_{2}\right)$.

Theorem B generalizes the result of Dorff [6, Theorem 5] who proved originally the same with $\gamma_{1}=\gamma_{2}=0$.

Generally, it is not easy to verify the local univalency of $f_{1} * f_{2}$ in $\mathbb{D}$. In [14], Li and Ponnusamy obtained the following result as a generalization of [6, Theorem 3].

Theorem C. Let $f=h+\bar{g} \in \mathcal{S}^{0}\left(H_{\gamma}\right)$ with the dilatation $\omega(z)=e^{i \theta} z^{n}$, where $n=1,2$ and $\theta \in \mathbb{R}$. Then $f_{0} * f \in \mathcal{S}_{H}^{0}$ and is convex in the direction $-\gamma$.

In 2010, Bshouty and Lyzzaik [3] brought out a collection of open problems and conjectures on planar harmonic mappings, proposed by many colleagues throughout the past quarter of a century. Dorff et. al (see [3, Problem 3.26]) posed the following open question.

Problem 1. (M. Dorff, M. Nowak and M. Woloszkiewicz)

(a) Let $f_{0}=h_{0}+\overline{g_{0}} \in \mathcal{S}^{0}\left(H_{0}\right)$ be as above with the dilatation $\omega_{0}=g_{0}^{\prime} / h_{0}^{\prime}=-z$, and $f=$ $h+\bar{g} \in \mathcal{S}\left(H_{0}\right)$ with the dilatation $\omega(z)=(z+a) /(1+a z), a \in(-1,1)$. Then $f_{0} * f \in S_{H}^{0}$ and is convex in the direction of the real axis. Determine the other values of $a \in \mathbb{D}$ for which the corresponding result holds.

(b) Let $f_{n} \in \mathcal{S}^{0}\left(H_{0}\right)$ with the dilatations $\omega_{n}(z)=e^{i \theta} z^{n}(\theta \in \mathbb{R}, n \in \mathbb{N})$. Determine the values of $n$ for which $f_{n} * f$ are univalent.

One of the proposers of the above problems communicated to the first author about a typo in [3. Problem 3.26(b)]. Again a typo in [3, Problem 3.26(a)] is now corrected and the corrected formulation is stated in Problem 1(b). Our primary aim in this paper is to present a solution to this open problem. However, Problem 1(a) has been solved by Li and Ponnusamy [13], and in [11, 14] the same has been solved in a more general setting which led to further investigation and interest in this topic. 
Note 1. In [8, Theorem 4], the following result was shown under the assumption that $f=h+\bar{g} \in \mathcal{K}_{H}^{0}$ with $h(z)+g(z)=z /(1-z)$ and $\omega(z)=\frac{z+a}{1+a z}$ with $a \in(-1,1)$. Unfortunately, the first condition gives $g^{\prime}(0)=0$ while the second condition gives $g^{\prime}(0)=a$. In view of this reasoning, we reformulate their result in the form which is needed in our proof.

Theorem 1.1. Let $f \in \mathcal{S}\left(H_{0}\right)$, i.e., $f=h+\bar{g} \in \mathcal{K}_{H}$ with $h(z)+g(z)=(1+a) \frac{z}{1-z}$, and $\omega(z)=\frac{z+a}{1+a z}$ with $a \in(-1,1)$. Then $f_{0} * f \in \mathcal{S}_{H}^{0}$ and is convex in the direction of the real axis.

Proof. In view of [6, Theorem A] and Lewy's Theorem, we just need to show that the dilatation $\widetilde{\omega}$ of $f_{0} * f$ satisfies the condition $|\widetilde{\omega}(z)|<1$ for all $z \in \mathbb{D}$. By assumption, we easily see that

$$
h^{\prime}(z)=\frac{1+a}{(1+\omega(z))(1-z)^{2}} \quad \text { and } \quad h^{\prime \prime}(z)=\frac{(1+a)\left[2(1+\omega(z))-\omega^{\prime}(z)(1-z)\right]}{(1+\omega(z))^{2}(1-z)^{3}}
$$

and the rest of the proof follows as in [8, Theorem 4]. For the sake of completeness we include the necessary details here. Indeed, since $g^{\prime}(z)=\omega(z) h^{\prime}(z)$, we have

$$
\begin{aligned}
\widetilde{\omega}(z) & =\frac{\left(g_{0} * g\right)^{\prime}(z)}{\left(h_{0} * h\right)^{\prime}(z)} \\
& =-z \frac{\omega^{\prime}(z) h^{\prime}(z)+\omega(z) h^{\prime \prime}(z)}{2 h^{\prime}(z)+z h^{\prime \prime}(z)} \\
& =-z \frac{p(z)}{p^{*}(z)}=-z \frac{(z+A)(z+B)}{(1+\bar{A} z)(1+\bar{B} z)},
\end{aligned}
$$

where

$$
p(z)=z^{2}+\frac{1+3 a}{2} z+\frac{1+a}{2}, \quad p^{*}(z)=z^{2} \overline{p(1 / \bar{z})}=1+\frac{1+3 a}{2} z+\frac{1+a}{2} z^{2},
$$

and $A, B$ are two zeros of $p(z)$. By using Cohn's rule,

$$
q_{1}(z)=\frac{\overline{a_{2}} p(z)-a_{0} p^{*}(z)}{z}=\frac{(a+3)(1-a)}{4}\left(z+\frac{1+3 a}{a+3}\right) .
$$

So $q_{1}(z)$ has one zero at $z_{0}=-\frac{1+3 a}{a+3}$ which is on the unit disk for $-1<a<1$. Thus, $|A|,|B|<1$.

\section{Main Results}

We now state and prove our main result which solves Problem 11(b) for $n=1$.

Theorem 2.1. Let $f=h+\bar{g} \in \mathcal{S}\left(H_{0}\right)$ with $h+g=(1+a) z /(1-z)$ and the dilatation $\omega(z)=$ $(z+a) /(1+a z)$, where $-1<a<1$, and $f_{1}=h_{1}+\overline{g_{1}} \in \mathcal{S}^{0}\left(H_{0}\right)$ with dilatation $\omega_{1}(z)=e^{i \theta} z(\theta \in \mathbb{R})$. Then $f_{1} * f$ is locally univalent and convex in the horizontal direction.

The problem of determining other values of $a \in \mathbb{D}$ and $|\epsilon|=1$ with $\omega(z)=\epsilon(z+a) /(1+a z)$ in Theorem 2.1 remains open. As in the case of [14, Theorem 1.3], establishing the analog of Theorem 2.1 for slanted half-plane mappings is another problem which needs further investigation. Note also that Theorem 2.1 for $\theta=\pi$ is contained in Theorem 1.1 .

To prove our main result, we need the following lemmas.

Lemma 2.2. ([4, 5.8 Corollary]) If $f=h+\bar{g}$ is convex, then $\left|\frac{g\left(z_{1}\right)-g\left(z_{2}\right)}{h\left(z_{1}\right)-h\left(z_{2}\right)}\right|<1$ for all $z_{1}, z_{2} \in \mathbb{D}$.

Lemma 2.3. ([17, Lemma 3]) Let $f: \mathbb{D} \rightarrow \mathbb{C}$ be nonconstant and analytic, where $f(\overline{\mathbb{D}})$ omits some point $w \in\{z: \operatorname{Re} z<0\}$. Suppose that $\widehat{f}\left(e^{i t}\right)=\lim _{z \rightarrow e^{i t}} f(z)$ exists for all $t \in \mathbb{R}$ (where possibly $\left.\widehat{f}\left(e^{i t}\right)=\infty\right)$. If $\operatorname{Re}\left\{\widehat{f}\left(e^{i t}\right)\right\} \geq 0$ for all t such that $\widehat{f}\left(e^{i t}\right)$ is finite, then $\operatorname{Re}\{f(z)\}>0$ for all $z \in \mathbb{D}$.

We remark that Lemma 2.3 is another convenient formulation of maximum modulus theorem for analytic functions. 
Proof of Theorem 2.1. Conclusion of Theorem 2.1 holds when $\theta=\pi$ (see Theorem 1.1) and thus, we will assume throughout the discussion that $\theta \neq \pi$. Now, by 1.2 and the assumption on $f$, we have

$$
h^{\prime}(z)+g^{\prime}(z)=\frac{1+a}{(1-z)^{2}} \text { and } \omega(z)=\frac{g^{\prime}(z)}{h^{\prime}(z)}=\frac{z+a}{1+a z} .
$$

Solving these two equations for $h^{\prime}$ and $g^{\prime}$ and then integrating the resulting equations give

$$
h(z)=\frac{1+a}{2} \frac{z}{1-z}+\frac{1-a}{4} \log \left(\frac{1+z}{1-z}\right) \text { and } g(z)=\frac{1+a}{2} \frac{z}{1-z}-\frac{1-a}{4} \log \left(\frac{1+z}{1-z}\right) .
$$

Again, by the assumption on $f_{1}$, we have

$$
h_{1}(z)+g_{1}(z)=\frac{z}{1-z} \text { and } \omega_{1}(z)=\frac{g_{1}^{\prime}(z)}{h_{1}^{\prime}(z)}=e^{i \theta} z .
$$

Solving these two equations gives

$$
\begin{aligned}
& h_{1}(z)=\frac{1}{1+e^{i \theta}} \frac{z}{1-z}+\frac{e^{i \theta}}{\left(1+e^{i \theta}\right)^{2}} \log \left(\frac{1+e^{i \theta} z}{1-z}\right), \text { and } \\
& g_{1}(z)=\frac{e^{i \theta}}{1+e^{i \theta}} \frac{z}{1-z}-\frac{e^{i \theta}}{\left(1+e^{i \theta}\right)^{2}} \log \left(\frac{1+e^{i \theta} z}{1-z}\right) .
\end{aligned}
$$

For any $F(z)=\sum_{n=1}^{\infty} c_{n} z^{n}$ analytic in $\mathbb{D}$, we see that $(z /(1-z)) * F(z)=F(z)$ and

$$
\log \left(\frac{1-x z}{1-z}\right) * F(z)=\int_{0}^{z} \frac{F(t)-F(x t)}{t} d t \quad \text { for }|x| \leq 1, x \neq 1 .
$$

In view of equations (2.1) and (2.2), a computation gives

$$
\begin{aligned}
h(z) * h_{1}(z)= & \frac{1+a}{2} h_{1}(z)+\frac{1-a}{4} \int_{0}^{z} \frac{h_{1}(t)-h_{1}(-t)}{t} d t \\
= & \frac{1+a}{2}\left[\frac{1}{\left(1+e^{i \theta}\right)} \frac{z}{1-z}+\frac{e^{i \theta}}{\left(1+e^{i \theta}\right)^{2}} \log \left(\frac{1+e^{i \theta} z}{1-z}\right)\right]+\frac{(1-a) e^{i \theta}}{4\left(1+e^{i \theta}\right)^{2}}\left[\operatorname{Li}_{2}(z)\right. \\
& \left.\quad-\operatorname{Li}_{2}(-z)+\operatorname{Li}_{2}\left(e^{i \theta} z\right)-\operatorname{Li}_{2}\left(-e^{i \theta} z\right)+\left(1+e^{-i \theta}\right) \log \left(\frac{1+z}{1-z}\right)\right]
\end{aligned}
$$

and

$$
\begin{aligned}
g(z) * g_{1}(z)= & \frac{1+a}{2} g_{1}(z)-\frac{1-a}{4} \int_{0}^{z} \frac{g_{1}(t)-g_{1}(-t)}{t} d t \\
= & \frac{1+a}{2}\left[\frac{e^{i \theta}}{\left(1+e^{i \theta}\right)} \frac{z}{1-z}-\frac{e^{i \theta}}{\left(1+e^{i \theta}\right)^{2}} \log \left(\frac{1+e^{i \theta} z}{1-z}\right)\right]+\frac{(1-a) e^{i \theta}}{4\left(1+e^{i \theta}\right)^{2}}\left[\operatorname{Li}_{2}(z)\right. \\
& \left.-\operatorname{Li}_{2}(-z)+\operatorname{Li}_{2}\left(e^{i \theta} z\right)-\operatorname{Li}_{2}\left(-e^{i \theta} z\right)-\left(1+e^{i \theta}\right) \log \left(\frac{1+z}{1-z}\right)\right],
\end{aligned}
$$

where $\operatorname{Li}_{2}(z)$ denotes the dilogarithm function defined by $\operatorname{Li}_{2}(z)=\sum_{k=1}^{\infty} \frac{z^{k}}{k^{2}}$ for $|z| \leq 1$.

Now the dilatation of $f * f_{1}$ is given by

$$
\widetilde{\omega}_{1}(z)=\frac{\left(g * g_{1}\right)^{\prime}(z)}{\left(h * h_{1}\right)^{\prime}(z)}=\frac{\frac{1+a}{2} z g_{1}^{\prime}(z)-\frac{1-a}{4}\left[g_{1}(z)-g_{1}(-z)\right]}{\frac{1+a}{2} z h_{1}^{\prime}(z)+\frac{1-a}{4}\left[h_{1}(z)-h_{1}(-z)\right]} .
$$

By Theorem B with $\gamma_{1}=0=\gamma_{2}$, we obtain that $f_{1} * f$ is convex in the horizontal direction provided $f_{1} * f$ is locally univalent in $\mathbb{D}$. Thus, it suffices to show that $\left|\widetilde{\omega}_{1}(z)\right|<1$ for $z \in \mathbb{D}$. In view of the last expression, $\left|\widetilde{\omega}_{1}(z)\right|<1$ is equivalent to

$$
\left|-\frac{1-a}{2(1+a)} \frac{g_{1}(z)-g_{1}(-z)}{z^{2} h_{1}^{\prime}(z)}+e^{i \theta}\right|^{2}|z|^{2}<\left|\frac{1-a}{2(1+a)} \frac{h_{1}(z)-h_{1}(-z)}{z h_{1}^{\prime}(z)}+1\right|^{2}
$$


and thus, it suffices to show

$$
\left|-\frac{1-a}{2(1+a)} \frac{g_{1}(z)-g_{1}(-z)}{z^{2} h_{1}^{\prime}(z)}+e^{i \theta}\right|^{2}<\left|\frac{1-a}{2(1+a)} \frac{h_{1}(z)-h_{1}(-z)}{z h_{1}^{\prime}(z)}+1\right|^{2},
$$

or equivalently,

$$
B(z):=\left|\frac{1-a}{2(1+a)} \frac{g_{1}(z)-g_{1}(-z)}{z^{2} h_{1}^{\prime}(z)}\right|^{2}-\left|\frac{1-a}{2(1+a)} \frac{h_{1}(z)-h_{1}(-z)}{z h_{1}^{\prime}(z)}\right|^{2}<\operatorname{Re}\left(\frac{1-a}{1+a} J(z)\right)
$$

where

$$
J(z)=\frac{h_{1}(z)-h_{1}(-z)}{z h_{1}^{\prime}(z)}+\frac{e^{-i \theta}\left(g_{1}(z)-g_{1}(-z)\right)}{z^{2} h_{1}^{\prime}(z)} .
$$

Because $h_{1}(0)=0=h_{1}^{\prime}(0)-1, g_{1}(0)=0=g_{1}^{\prime}(0)$ and $h_{1}^{\prime}(z) \neq 0$ on $\mathbb{D}$, the function $J(z)$ is clearly analytic on $\mathbb{D}$. Since $f_{1}$ is convex, it follows from Lemma 2.2 that

$$
\left|\frac{g_{1}(z)-g_{1}(-z)}{h_{1}(z)-h_{1}(-z)}\right|<1 \text {. }
$$

Also, since

$$
\lim _{z \rightarrow 0} \frac{g_{1}(z)-g_{1}(-z)}{h_{1}(z)-h_{1}(-z)}=\lim _{z \rightarrow 0} \frac{g_{1}^{\prime}(z)+g_{1}^{\prime}(-z)}{h_{1}^{\prime}(z)+h_{1}^{\prime}(-z)}=0,
$$

by Schwarz's Lemma, we conclude that

$$
\left|\frac{g_{1}(z)-g_{1}(-z)}{h_{1}(z)-h_{1}(-z)}\right|<|z|
$$

which clearly implies $B(z)<0$ for $z \in \mathbb{D}$, where $B(z)$ is given by (2.4). Thus, by (2.4), the proof is complete if we show that $\operatorname{Re}\{J(z)\}>0$ in $\mathbb{D}$. In order to do this, by $(2.2)$, we need to simplify the expression for $J(z)$ as

$$
J(z)=\frac{\left(1+e^{i \theta} z\right)(1-z)}{\left(1+e^{i \theta}\right) z}\left[2-\frac{(1-z)\left(1-e^{i \theta} z\right)}{\left(1+e^{i \theta}\right) z}\left\{\log \left(\frac{1+e^{i \theta} z}{1-z}\right)-\log \left(\frac{1-e^{i \theta} z}{1+z}\right)\right\}\right] .
$$

By Lemma 2.3, we need to check that $\lim _{z \rightarrow e^{i t}} J(z)$ exists for all $t \in \mathbb{R}$ (where possibly $J\left(e^{i t}\right)=\infty$ ). This is clearly the case for all $t \in \mathbb{R} \backslash\{0, \pi-\theta, \pi, 2 \pi-\theta\}$. For the remaining values of $t$, we obtain the following limits:

$$
\lim _{z \rightarrow 1} J(z)=0, \quad \lim _{z \rightarrow-1} J(z)=\left\{\begin{array}{rl}
0 & \text { if } \theta=0 \\
\infty & \text { if } \theta \neq 0
\end{array}, \quad \lim _{z \rightarrow e^{i(\pi-\theta)}} J(z)=0,\right.
$$

and

$$
\lim _{z \rightarrow e^{i(2 \pi-\theta)}} J(z)=4 i \tan \frac{\theta}{2} \text {. }
$$

Consequently, it suffices to show that $\operatorname{Re}\left\{J\left(e^{i t}\right)\right\} \geq 0$ for all $t \in \mathbb{R} \backslash\{0, \pi-\theta, \pi, 2 \pi-\theta\}$. We have

$$
\begin{aligned}
J\left(e^{i t}\right)= & \frac{\left(1+e^{i(\theta+t)}\right)\left(1-e^{i t}\right)}{\left(1+e^{i \theta}\right) e^{i t}}\left[2-\frac{\left(1-e^{i t}\right)\left(1-e^{i(\theta+t)}\right)}{\left(1+e^{i \theta}\right) e^{i t}}\left\{\log \left(\frac{1+e^{i(\theta+t)}}{1-e^{i t}}\right)\right.\right. \\
\left.\left.-\log \left(\frac{1-e^{i(\theta+t)}}{1+e^{i t}}\right)\right\}\right] & \\
= & \frac{-2 i \sin \frac{t}{2} \cos \frac{\theta+t}{2}}{\cos \frac{\theta}{2}}\left[2+\frac{2 \sin \frac{t}{2} \sin \frac{\theta+t}{2}}{\cos \frac{\theta}{2}}\left\{\log \left(\frac{1+e^{i(\theta+t)}}{1-e^{i t}}\right)-\log \left(\frac{1-e^{i(\theta+t)}}{1+e^{i t}}\right)\right\}\right]
\end{aligned}
$$

and hence,

$$
\operatorname{Re}\left\{J\left(e^{i t}\right)\right\}=\frac{2 \sin ^{2} \frac{t}{2} \sin (\theta+t)}{\cos ^{2} \frac{\theta}{2}}\left[\arg \left(\frac{1+e^{i(\theta+t)}}{1-e^{i t}}\right)-\arg \left(\frac{1-e^{i(\theta+t)}}{1+e^{i t}}\right)\right] .
$$


We now introduce

$$
A=\arg \left(\frac{1+e^{i(\theta+t)}}{1-e^{i t}}\right) \text { and } B=\arg \left(\frac{1-e^{i(\theta+t)}}{1+e^{i t}}\right)
$$

so that for $0 \leq \theta<\pi$, we have

$$
A=\left\{\begin{array}{ll}
\frac{\theta+\pi}{\frac{2}{2}} & \text { if } \mathrm{t} \in(0, \pi-\theta) \\
\frac{\theta-\pi}{2} & \text { if } \mathrm{t} \in(\pi-\theta, 2 \pi)
\end{array}, \quad \text { and } B= \begin{cases}\frac{\theta-\pi}{2} & \text { if } \mathrm{t} \in(0, \pi) \cup(2 \pi-\theta, 2 \pi) \\
\frac{\theta+\pi}{2} & \text { if } \mathrm{t} \in(\pi, 2 \pi-\theta) .\end{cases}\right.
$$

Then

$$
A-B=\left\{\begin{aligned}
\pi & \text { if } \mathrm{t} \in(0, \pi-\theta) \\
-\pi & \text { if } \mathrm{t} \in(\pi, 2 \pi-\theta) \\
0 & \text { if } \mathrm{t} \in(\pi-\theta, \pi) \cup(2 \pi-\theta, 2 \pi) .
\end{aligned}\right.
$$

Next, we consider $\sin (\theta+t)$. It is non-negative when $t \in(0, \pi-\theta) \cup(2 \pi-\theta, 2 \pi)$, and negative when $t \in(\pi-\theta, 2 \pi-\theta)$. This observation shows that $\operatorname{Re}\left\{J\left(e^{i t}\right)\right\} \geq 0$ for all cases.

The general result for $\theta \in(-\pi, \pi)$ is similar to the above discussion for the case $\theta \in[0, \pi)$. This completes the proof of the assertion $\left|\widetilde{\omega}_{1}(z)\right|<1$ in $\mathbb{D}$.

The images of the unit disk $\mathbb{D}$ under $f * f_{1}$ for $\theta=\pi / 6$ and $a=-0.5,0,0.5,0.8$ are shown in Figure 1(a)-(d). The images of $\mathbb{D}$ under $f * f_{1}$ for $a=0.5$ and $\theta=0, \pi / 6, \pi / 3, \pi / 2$ are shown in Figure 2(a)-(d). We plot these images as equally spaced concentric radial segments.

\section{The Dilatations of $f * f_{n}$}

Now we compute the formulas for the dilatations of $f * f_{n}$, where $f=h+\bar{g} \in \mathcal{S}\left(H_{0}\right)$ with $h+g=(1+a) z /(1-z)$ and the dilatation $\omega(z)=(z+a) /(1+a z)$, where $-1<a<1$, and $f_{n} \in \mathcal{S}^{0}\left(H_{0}\right)$ with the dilatation $\omega_{n}(z)=e^{i \theta} z^{n}(\theta \in \mathbb{R}, n \in \mathbb{N})$. First, we begin by computing the representation of $f_{n}$ when $\theta=\pi$. Note that in this case

and thus,

$$
h_{n}^{\prime}(z)+g_{n}^{\prime}(z)=\frac{1}{(1-z)^{2}} \quad \text { and } \quad g_{n}^{\prime}(z)=-z^{n} h_{n}^{\prime}(z)
$$

In order to compute $h_{n}(z)$, we may rewrite it as

$$
h_{n}^{\prime}(z)=\frac{1}{\left(1-z^{n}\right)(1-z)^{2}} .
$$

$$
h_{n}^{\prime}(z)=\frac{n^{2}-1}{12 n} \frac{1}{1-z}+\frac{n-1}{2 n} \frac{1}{(1-z)^{2}}+\frac{1}{n} \frac{1}{(1-z)^{3}}+\frac{1}{n} \sum_{k=1}^{n-1} \frac{1}{\left(1-e^{\frac{2 k \pi}{n}} i\right)^{2}} \frac{1}{\left(1-z e^{-\frac{2 k \pi}{n} i}\right)} .
$$

By integrating the previous expression we arrive at

$$
\begin{aligned}
& h_{n}(z)=\frac{n-1}{2 n} \frac{z}{1-z}+\frac{1}{2 n} \frac{z(2-z)}{(1-z)^{2}}-\frac{n^{2}-1}{12 n} \log (1-z)+\frac{1}{4 n} \sum_{k=1}^{n-1} \csc ^{2} \frac{\pi k}{n} \log \left(1-z e^{-\frac{2 k \pi}{n} i}\right), \\
& g_{n}(z)=\frac{n+1}{2 n} \frac{z}{1-z}-\frac{1}{2 n} \frac{z(2-z)}{(1-z)^{2}}+\frac{n^{2}-1}{12 n} \log (1-z)-\frac{1}{4 n} \sum_{k=1}^{n-1} \csc ^{2} \frac{\pi k}{n} \log \left(1-z e^{-\frac{2 k \pi}{n} i}\right) .
\end{aligned}
$$

From 2.1 and (2.3), we obtain $f * f_{n}$ from

$$
\begin{aligned}
& h(z) * h_{n}(z)=\frac{1-a}{4} \int \frac{h_{n}(z)-h_{n}(-z)}{z} d z+\frac{1+a}{2} h_{n}(z), \\
& g(z) * g_{n}(z)=-\frac{1-a}{4} \int \frac{g_{n}(z)-g_{n}(-z)}{z} d z+\frac{1+a}{2} g_{n}(z),
\end{aligned}
$$




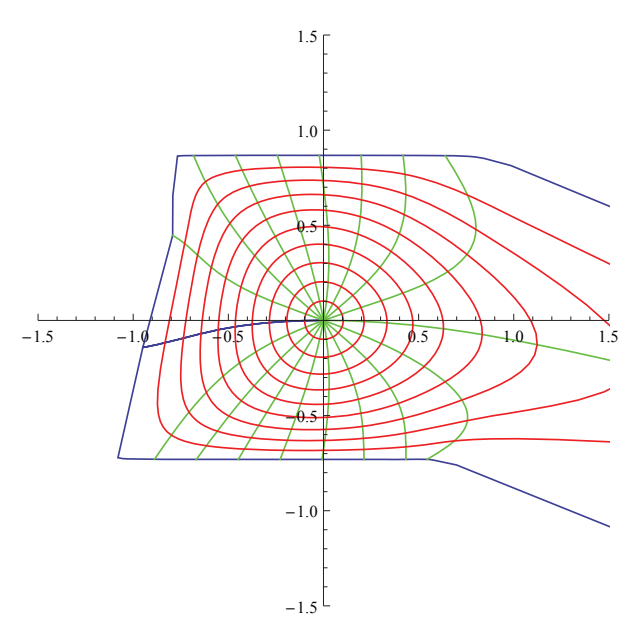

(a) $a=-0.5$

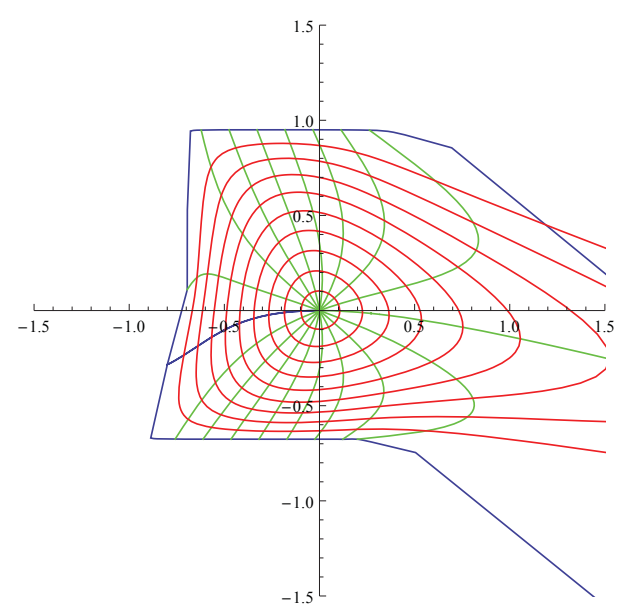

(c) $a=0.5$

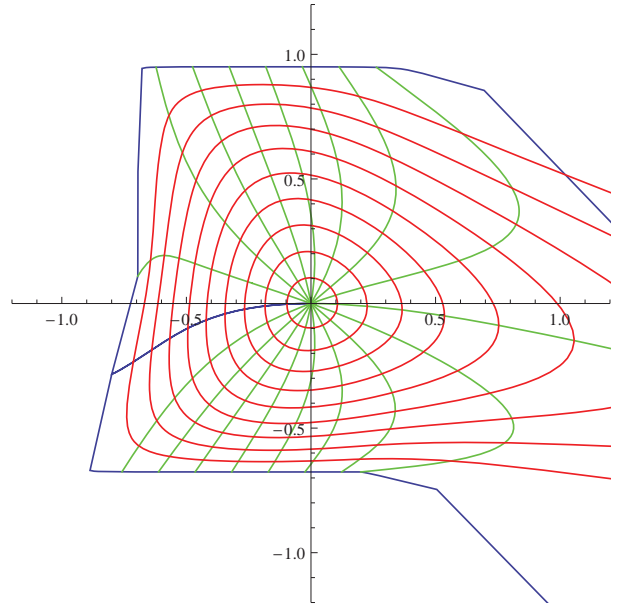

(b) $a=0$

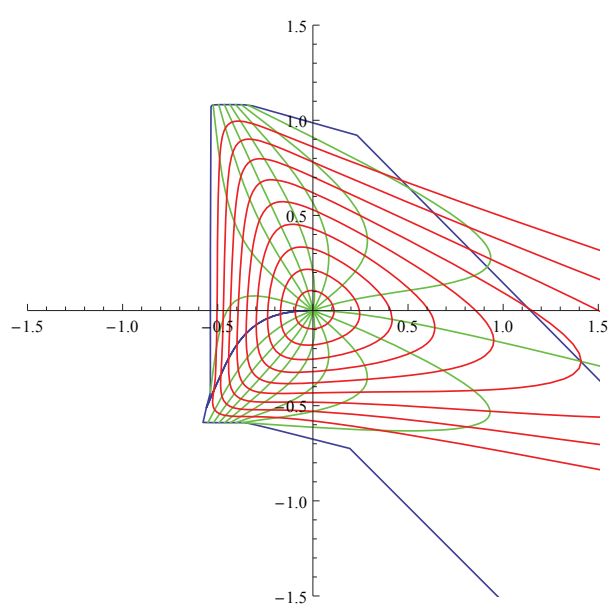

(d) $a=0.8$

Figure 1. Images of $\mathbb{D}$ under $f * f_{1}$ for $\theta=\pi / 6$ and for certain values of $a$.

and the dilatation $\widetilde{\omega}_{n}$ of $f * f_{n}$ is then given by

$$
\widetilde{\omega}_{n}(z)=\frac{\left(g * g_{n}\right)^{\prime}(z)}{\left(h * h_{n}\right)^{\prime}(z)}=\frac{-\frac{1-a}{4}\left(g_{n}(z)-g_{n}(-z)\right)+\frac{1+a}{2} z g_{n}^{\prime}(z)}{\frac{1-a}{4}\left(h_{n}(z)-h_{n}(-z)\right)+\frac{1+a}{2} z h_{n}^{\prime}(z)} .
$$

Table 1 presents values of $\left|\widetilde{\omega}_{n}(z)\right|$ for certain choices of $n, a$ and $z$ with the help of Mathematica. From Table 1, we observe for some $n \geq 2$ that $\left|\widetilde{\omega}_{n}(z)\right|>1$ for certain values of $a$ and $z$.

Next, we assume that $\theta \neq \pi$ and consider

$$
h_{n}^{\prime}(z)+g_{n}^{\prime}(z)=\frac{1}{(1-z)^{2}} \quad \text { and } \quad \omega_{n}(z)=\frac{g_{n}^{\prime}(z)}{h_{n}^{\prime}(z)}=e^{i \theta} z^{n}
$$




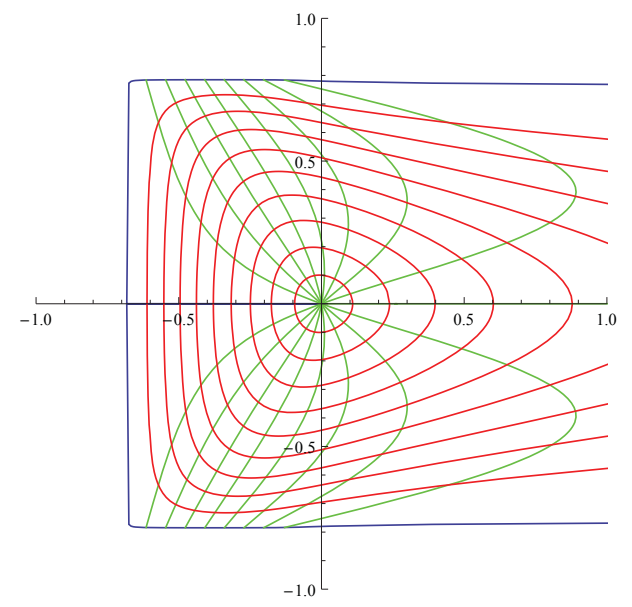

(a) $\theta=0$

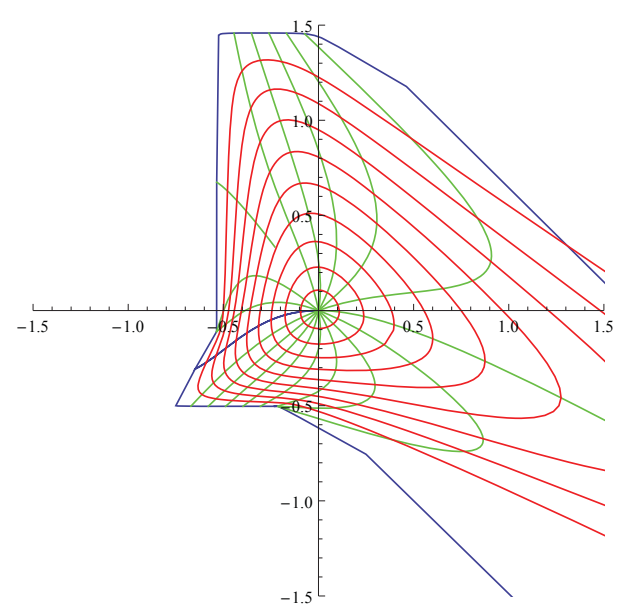

(c) $\theta=\pi / 3$

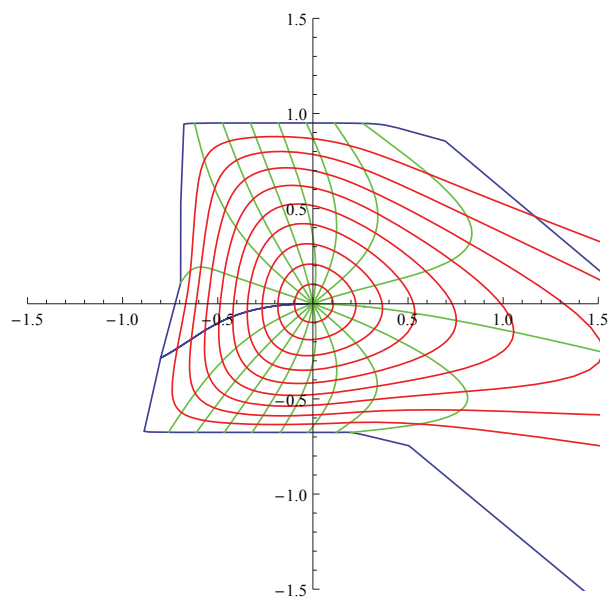

(b) $\theta=\pi / 6$

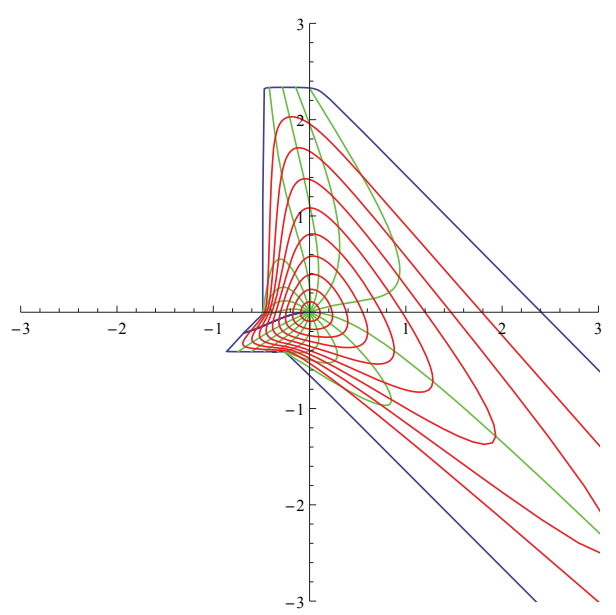

(d) $\theta=\pi / 2$

Figure 2. Images of $\mathbb{D}$ under $f * f_{1}$ for $a=0.5$ and for certain values $\theta$.

so that

$$
\begin{aligned}
h_{n}^{\prime}(z)= & \frac{1}{\left(1+e^{i \theta} z^{n}\right)(1-z)^{2}} \\
= & \frac{n e^{i \theta}}{\left(1+e^{i \theta}\right)^{2}} \frac{1}{1-z}+\frac{1}{1+e^{i \theta}} \frac{1}{(1-z)^{2}} \\
& -\frac{1}{n} \sum_{k=0}^{n-1} \frac{1}{\left(1-e^{i \frac{(2 k+1) \pi-\theta}{n}}\right)^{2}} \frac{1}{1-z e^{-i \frac{(2 k+1) \pi-\theta}{n}}} .
\end{aligned}
$$

Integration gives

$$
\begin{aligned}
h_{n}(z)= & -\frac{n e^{i \theta}}{\left(1+e^{i \theta}\right)^{2}} \log (1-z)+\frac{1}{1+e^{i \theta}} \frac{z}{1-z} \\
& +\frac{1}{4 n} \sum_{k=0}^{n-1} \csc ^{2} \frac{(2 k+1) \pi-\theta}{2 n} \log \left(1-z e^{-i \frac{(2 k+1) \pi-\theta}{n}}\right),
\end{aligned}
$$




\begin{tabular}{|c|c|c|c|}
\hline \hline$n$ & $a$ & $z$ & $\left|\widetilde{\omega}_{n}(z)\right|$ \\
\hline 2 & 0.5 & $0.99 \exp \left(\frac{\pi}{3} i\right)$ & 1.06019 \\
\hline 3 & 0.5 & $0.99 \exp \left(\frac{3 \pi}{4} i\right)$ & 1.28884 \\
\hline 4 & -0.5 & $0.99 \exp \left(\frac{\pi}{8} i\right)$ & 1.07326 \\
\hline 5 & -0.5 & $0.99 \exp \left(\frac{\pi}{10} i\right)$ & 1.04422 \\
\hline 6 & -0.4 & $0.99 \exp \left(\frac{\pi}{11} i\right)$ & 1.03038 \\
\hline 7 & 0.5 & $0.99 \exp \left(\frac{\pi}{3} i\right)$ & 1.04396 \\
\hline 8 & 0.5 & $0.99 \exp \left(\frac{\pi}{3} i\right)$ & 1.02052 \\
\hline 9 & 0.5 & $0.99 \exp \left(\frac{\pi}{2} i\right)$ & 1.12641 \\
\hline 10 & 0.3 & $0.99 \exp \left(\frac{\pi}{4} i\right)$ & 1.05563 \\
\hline 11 & -0.7 & $0.99 \exp \left(\frac{\pi}{5} i\right)$ & 1.32055 \\
\hline 12 & 0 & $0.99 \exp \left(\frac{\pi}{5} i\right)$ & 1.09197 \\
\hline 13 & 0 & $0.99 \exp \left(\frac{\pi}{5} i\right)$ & 1.00698 \\
\hline 14 & -0.4 & $0.99 \exp \left(\frac{\pi}{6} i\right)$ & 1.20222 \\
\hline 15 & -0.2 & $0.99 \exp \left(\frac{\pi}{6} i\right)$ & 1.04876 \\
\hline
\end{tabular}

TABLE 1. Values of $\left|\widetilde{\omega}_{n}(z)\right|$ for certain values $a$ and $z$.

and thus

$$
\begin{aligned}
g_{n}(z)= & \frac{z}{1-z}-h_{n}(z) \\
= & \frac{n e^{i \theta}}{\left(1+e^{i \theta}\right)^{2}} \log (1-z)+\frac{e^{i \theta}}{1+e^{i \theta}} \frac{z}{1-z} \\
& -\frac{1}{4 n} \sum_{k=0}^{n-1} \csc ^{2} \frac{(2 k+1) \pi-\theta}{2 n} \log \left(1-z e^{-i \frac{(2 k+1) \pi-\theta}{n}}\right) .
\end{aligned}
$$

Substituting the expressions $(3.4)$ and $(3.5)$ into 3.3 , we get Table 2 .

\begin{tabular}{|c|c|c|c|c|}
\hline$n$ & $a$ & $\theta$ & $z$ & $\left|\widetilde{\omega}_{n}(z)\right|$ \\
\hline 2 & 0.5 & $\frac{\pi}{8}$ & $0.99 \exp \left(\frac{\pi}{2} i\right)$ & 1.16334 \\
\hline 3 & 0.5 & $\frac{\pi}{12}$ & $0.99 \exp \left(\frac{\pi}{2} i\right)$ & 1.09124 \\
\hline 4 & 0.5 & $\frac{\pi}{3}$ & $0.99 \exp \left(\frac{\pi}{3} i\right)$ & 1.05616 \\
\hline 5 & 0.8 & $\frac{\pi}{6}$ & $0.99 \exp \left(\frac{2 \pi}{3} i\right)$ & 1.06377 \\
\hline 6 & 0.7 & $\frac{\pi}{3}$ & $0.99 \exp \left(\frac{\pi}{2} i\right)$ & 1.09271 \\
\hline 7 & 0.7 & $\frac{\pi}{6}$ & $0.99 \exp \left(\frac{\pi}{2} i\right)$ & 1.01364 \\
\hline 8 & 0.6 & $-\frac{\pi}{3}$ & $0.99 \exp \left(\frac{\pi}{2} i\right)$ & 1.04091 \\
\hline 9 & 0.7 & $\frac{\pi}{2}$ & $0.99 \exp \left(-\frac{7 \pi}{8} i\right)$ & 1.20496 \\
\hline 10 & 0.7 & $-\frac{\pi}{2}$ & $0.99 \exp \left(-\frac{7 \pi}{8} i\right)$ & 1.97405 \\
\hline 11 & 0.4 & $\frac{\pi}{2}$ & $0.99 \exp \left(-\frac{7 \pi}{8} i\right)$ & 1.42585 \\
\hline 12 & 0 & $\frac{\pi}{2}$ & $0.99 \exp \left(\frac{7 \pi}{8} i\right)$ & 1.09957 \\
\hline 13 & 0.9 & $-\frac{\pi}{16}$ & $0.99 \exp \left(\frac{7 \pi}{8} i\right)$ & 1.01078 \\
\hline 14 & 0.9 & $-\frac{3 \pi}{4}$ & $0.99 \exp \left(-\frac{7 \pi}{8} i\right)$ & 1.08478 \\
\hline 15 & 0.9 & $-\frac{\pi}{4}$ & $0.99 \exp \left(\frac{7 \pi}{8} i\right)$ & 1.00032 \\
\hline
\end{tabular}

TABLE 2. Values of $\left|\widetilde{\omega}_{n}(z)\right|$ for certain values $a, \theta$ and $z$. 
From the above discussion and some complicated calculations and experiments, we propose the following.

Problem 2. Let $f=h+\bar{g} \in \mathcal{S}\left(H_{0}\right)$ with $h+g=(1+a) z /(1-z)$ and the dilatation $\omega(z)=$ $(z+a) /(1+a z)$, where $-1<a<1$, and $f_{n}=h_{n}+\overline{g_{n}} \in \mathcal{S}^{0}\left(H_{0}\right)$ with dilatations $\omega_{n}(z)=e^{i \theta} z^{n}(\theta \in$ $\mathbb{R}, n \in \mathbb{N}$ ). We conjecture that $f * f_{n}$ is not locally univalent for $n \geq 2$. In this situation, it is natural to ask for the radius of univalency of $f * f_{n}$.

Acknowledgements. The authors thank the referee for pointing out the error occurred in Problem 1 in page 2, and the comments which essentially improved the article. The research of the first author was supported by the Project Education Fund of Yunnan Province under Grant No. 2015Y456, the First Bath of Young and Middle-aged Academic Training Object Backbone of Honghe University under Grant No. 2014GG0102. The work of the first author was completed during his visit to Indian Statistical Institute, Chennai Centre.

\section{REFERENCES}

[1] Y. Abu-Muhanna and G. Schober, Harmonic mappings onto convex domains, Can. J. Math. 39 (1987), 1489-1530.

[2] Z. Boyd, M. Dorff, M. Nowak, M. Romney and M. Wołoszkiewicz, Univalency of convolutions of harmonic mappings, Appl. Comput. Math. 234 (2014), 326-332.

[3] D. Bshouty and A. Lyzzaik, Problems and conjectures in planar harmonic mappings, In: Proc. ICM2010 Satellite Conf. Int. Workshop on Harmonic and Quasiconformal Mappings, IIT Madras, Aug. 09-17, 2010, ed. by D. Minda, S. Ponnusamy, and N. Shanmugalingam, J. Analysis 18(2010), 69-81.

[4] J. Clunie and T. Sheil-Small, Harmonic univalent functions, Ann. Acad. Sci. Fenn. Ser. A.I. Math. 9 (1984), 3-25.

[5] M. Dorff, Harmonic mappings onto asymmetric vertical strips, in Computational Methods and Function Theorey 1997, (N. Papamichael, St. Ruscheweyh and E. B. Staff. eds.), 171-175, World Sci. Publishing, River Edge, NJ, 1999.

[6] M. Dorff, Convolutions of planar harmonic convex mappings, Comp. Var. Theorey Appl. 45(3)(2001), 263-271.

[7] M. Dorff, Anamorphosis, mapping problems, and harmonic univalent functions, chapter in Explorations in Complex Analysis, Mathematical Association of America, 2012.

[8] M. Dorff, M. Nowak and M. Wołoszkiewicz, Convolutions of harmonic convex mappings, Complex Var. Elliptic Equ. 57(5)(2012), 489-503.

[9] P. Duren, Harmonic mappings in the plane, Cambridge Univ. Press, 2004.

[10] W. Hengartner and G. Schober, Univalent harmonic functions, Trans. Amer. Math. Soc. 299 (1987), 1-31.

[11] Y. Jiang, A. Rasila, and Y. Sun, A note on convexity of convolutions of harmonic mappings, Bull. Korean Math. Soc. 52(6) (2015), 1925-1935.

[12] H. Lewy, On the non-vanishing of the Jacobian in certain one-to-one mappings, Bull. Amer. Math. Soc. 42(1936), 689-692.

[13] L. Li and S. Ponnusamy, Solution to an open problem on convolutions of harmonic mappings, Complex Var. Elliptic Equ. 58(12) (2013), 1647-1653.

[14] L. Li and S. Ponnusamy, Convolutions of slanted half-plane harmonic mappings, Analysis (Munich). 33(2) (2013), 159-176.

[15] Z. Liu and Y. Li, Convolutions of generalized harmonic half-plane mappings with harmonic strip mappings. Abstr. Appl. Anal. 2014, Article ID 474239.

[16] S. Ponnusamy and A. Rasila, Planar harmonic and quasiregular mappings, Topics in Modern Function Theory (Editors. St. Ruscheweyh and S. Ponnusamy): Chapter in CMFT, RMSLecture Notes Series 19 (2013), 267-333. 
[17] M. Romney, A class of univalent convolutions of harmonic mappings, (2013). All Theses and Dissertations. Paper 4169.

[18] Z. Wang, Z. Liu and Y. Li, On convolutions of harmonic univalent mappings convex in the direction of the real axis, J. Appl. Anal. Comput. 6(1) (2016), 145-155.

Z.Liu, School of Mathematics and Econometrics, Hunan University, Changsha 410082, Hunan, PeoPLE'S Republic of China.

College of Mathematics, Honghe University, Mengzi 661199, Yunnan, People's Republic of China. E-mail address: liuzhihongmath@163.com

S. Ponnusamy, Indian Statistical Institute (ISI), Chennai Centre, Sets (Society for Electronic Transactions and Security), MGR Knowledge City, Cit Campus, Taramani, Chennai 600 113, India.

E-mail address: samy@isichennai.res.in, samy@iitm.ac.in 